\title{
Physics of high-power InGaN/GaN lasers
}

\author{
J. Piprek and S. Nakamura
}

\begin{abstract}
The authors analyse the performance and device physics of nitride laser diodes that exhibit the highest room-temperature continuous-wave output power. The analysis is based on advanced laser simulation. The laser model self-consistently combines band structure and freecarrier gain calculations with two-dimensional simulations of wave guiding, carrier transport and heat flux. Material parameters used in the model are carefully evaluated. Excellent agreement between simulations and measurements is achieved. The maximum output power is limited by electron leakage into the p-doped ridge. Leakage escalation is caused by strong self-heating, gain reduction and elevated carrier density within the quantum wells. Built-in polarisation fields are found to be effectively screened at high-power operation. Improved heat-sinking is predicted to allow for a significant increase of the maximum output power.
\end{abstract}

\section{Introduction}

We investigate Fabry-Perot laser diodes with two InGaN/GaN quantum wells (QWs) as previously fabricated [1]. Those devices exhibit the highest output power $(420 \mathrm{~mW})$ reported thus far for room-temperature continuous-wave operation of nitride lasers. The active region includes a p-doped $\mathrm{AlGaN}$ electron stopper layer (Fig. 1). It is sandwiched between GaN separate confinement layers and $\mathrm{AlGaN} / \mathrm{GaN}$ superlattice (SL) cladding layers (Table 1). The thickness of the n-side cladding layer was increased from $600 \mathrm{~nm}$ to $1200 \mathrm{~nm}$ to reduce the penetration of the laser light into the GaN substrate. For optical and current confinement, a $3 \mu \mathrm{m}$ wide ridge is etched into the top two layers. The cleaved front facet has a power reflectivity of $R_{f}=0.18$ and the back facet is coated with two periods of $\mathrm{SiO}_{2} / \mathrm{TiO}_{2}$ for high reflectivity $\left(R_{b}=0.95\right)$. Our example device is $450 \mu \mathrm{m}$ long.

Advanced laser simulation is used to analyse internal physical processes of this laser diode. We utilise commercially available laser simulation software [2] to reveal performance limiting mechanisms and to explore design optimisation options.

\section{Laser model and material parameters}

The laser model self-consistently combines band structure and free-carrier gain calculations with two-dimensional (2-D) simulations of wave guiding, carrier transport and heat flux. Details of the laser model are available on the Internet

\footnotetext{
(C) IEE, 2002

IEE Proceedings online no. 20020441

DOI: 10.1049 /ip-opt:20020441

Paper first received 3rd December 2001 and in revised form 19th April 2002

J. Piprek is with the Electrical and Computer Engineering Department, University of California, Santa Barbara, CA 93106, USA

S. Nakamura is with the Materials Department, University of California, Santa Barbara, CA 93106, USA
}

[Note 1]; we here discuss only those aspects that are crucial to our analysis.

The carrier drift-diffusion model includes Fermi statistics and incomplete ionisation of dopants. The superlattice is treated as uniform material of average composition. The high activation energy of the $\mathrm{Mg}$ acceptor causes the hole density to be considerably smaller than the $\mathrm{Mg}$ density. We use an $\mathrm{Mg}$ activation energy of $170 \mathrm{meV}$ for $\mathrm{GaN}$ [3] which is assumed to increase by $3 \mathrm{meV}$ per \% Al for AlGaN. Measured hole mobilities $\mu_{p}$ are employed (Table 1). In n-type material, the $\mathrm{Si}$ donor activation energy is $\sim 20 \mathrm{meV}$ [4]. Room temperature electron mobilities up to $\mu_{n}=900 \mathrm{~cm}^{2} / \mathrm{V}$ s have been measured for lowdoped GaN, which are reduced by more than one order of magnitude in highly n-doped GaN [5]. Monte Carlo simulations of various nitride alloys have recently been used to establish analytical expressions for the electron mobility as a function of doping density [6] from which the values in Table 1 are extracted. The influence of the electric field on the mobilities of electrons and holes is illustrated in Fig. 2. Mobility reduction with temperature elevation is considered as $\mu_{n} \propto T^{-1.5}$ [4] and $\mu_{p} \propto T^{-2}$ (estimation based on [7]).

Defect related recombination is known to be the main carrier loss mechanism in nitride devices. The ShockleyRead-Hall (SRH) recombination lifetime of electrons and holes is assumed to be $1 \mathrm{~ns}$; however, this is a rough estimate since the type and density of recombination centres are sensitive to the technological process. Within passive layers, a spontaneous emission parameter of $B=$ $2 \times 10^{-11} \mathrm{~cm}^{3} \mathrm{~s}^{-1}$ is employed [8]. The spontaneous recombination rate in quantum wells is larger than in passive layers and it is calculated by integration of the spontaneous emission spectrum. From its bandgap dependence in other materials, a very small Auger parameter of $C=10^{-34} \mathrm{~cm}^{6} \mathrm{~s}^{-1}$ is estimated for GaN. Thus, even with large carrier densities, Auger recombination in nitride materials is negligible.

A wide spectrum of bandgap bowing parameters has been obtained for ternary nitride alloys due to differences

Note 1: http://www.crosslight.com 


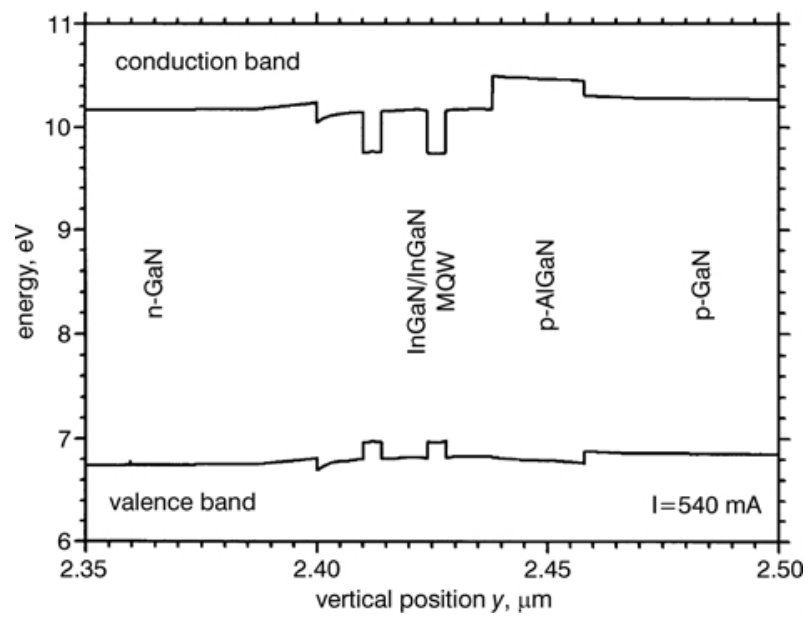

Fig. 1 Band diagram of active region

in growth and measurement conditions [9, 10]. For unstrained layers with low mole fraction of the alloy element $(x<0.2)$, we employ the following roomtemperature relations for the direct bandgap $[\mathrm{eV}]$ :

$$
\begin{aligned}
& E_{g}\left(\operatorname{In}_{x} \mathrm{Ga}_{1-x} \mathrm{~N}\right)=1.89 x+3.42(1-x)-3.8 x(1-x) \\
& E_{g}\left(\mathrm{Al}_{x} \mathrm{Ga}_{1-x} \mathrm{~N}\right)=6.28 x+3.42(1-x)-1.3 x(1-x)
\end{aligned}
$$

A band offset ratio of $\Delta E_{c} / \Delta E_{v}=0.7 / 0.3$ is assumed for $\mathrm{InGaN} / \mathrm{GaN}$ as well as for AlGaN/GaN, which corresponds to an average of reported values for each case [11, 12] (Fig. 1).

In our strained InGaN quantum wells, the conduction bands are assumed to be parabolic and the nonparabolic valence bands are computed by a $6 \times 6 \mathrm{kp}$ method [13]. Binary effective mass parameters, lattice constants and elastic constants from Table 2 are linearly interpolated to obtain InGaN values. GaN values are used for the deformation potentials [14]. Calculated valence bands are shown in Fig. 3 illustrating strong valence band mixing. Similar band structures have been calculated by other authors [15]. For such nitride layers, grown along the $c$-axis, the quantum well strain is biaxial, lowering the crystal-field splithole $(\mathrm{CH})$ band but hardly separating heavy hole $(\mathrm{HH})$ and light hole $(\mathrm{LH})$ bands, i.e. strain is not as effective in reducing the threshold carrier density as in GaAs or
InP based lasers. Uniaxial strain promises lower threshold currents [16]. Thermal bandgap shrinkage is considered with $d E_{g} / d T=-0.6 \mathrm{meV} / \mathrm{K}$ [17]. Bandgap renormalisation caused by carrier-carrier interaction depends on the quantum well 2-D carrier density as $\Delta E_{g}=-\zeta n_{2 D}^{1 / 3}$ with $\zeta=6 \times 10^{-6} \mathrm{eV} \mathrm{cm}^{2 / 3}$ being independent of well thickness and nitride composition [18].

The optical gain mechanism in InGaN quantum wells of real lasers is still not fully understood. It may be strongly affected by a nonuniform In distribution. Internal polarisation fields tend to separate quantum confined electrons and holes, thereby reducing optical gain and spontaneous emission. However, screening by electrons and holes is expected to suppress QW polarisation fields at high current operation [19]. The high carrier density is also assumed to eliminate exciton effects, despite the large exciton binding energy in nitrides. On the other hand, many-body models predict significant gain enhancement at high carrier densities [20]. Considering all the uncertainties in calculating the gain of our quantum wells, we here start with a simple free carrier gain model, including a Lorentzian broadening function with $0.1 \mathrm{ps}$ scattering time [21]. Rectangular quantum wells are assumed. The resulting gain characteristic is plotted in Fig. 4 for different temperatures.

Optical reflection and waveguiding mainly depends on the refractive index profile inside the device. For photon energies close to the bandgap, the refractive index is a strong function of wavelength. For the design of optical waveguides, the compositional change of the refractive index is often more important than its absolute value. In comparison to refractive index measurements on $\mathrm{Al}_{x} \mathrm{Ga}_{1-x} \mathrm{~N}(x<0.38)$, the Adachi model [22] was found to be most accurate using fit parameters obtained from measurements [23]. Reliable refractive index measurements on $\mathrm{In}_{x} \mathrm{Ga}_{1-x} \mathrm{~N}$ are currently not available so that a linear interpolation of binary parameters is chosen here [24]. In addition, we employ the bandgap relations given in (1) and (2), respectively. The resulting refractive index values are given in Table 1. The vertical index profile of our laser is plotted in Fig. 5, together with the optical intensity.

Measurements of the thermal conductivity are hardly published for nitride compounds. For GaN, a roomtemperature value of $1.3 \mathrm{~W} / \mathrm{cm} \mathrm{K}$ is reported in [25]. All other thermal conductivities in Table 1 are estimated, considering the impact of alloy and interface scattering

\begin{tabular}{|c|c|c|c|c|c|}
\hline Parameter & $I(\mathrm{~nm})$ & $N_{\text {dop }}\left(1 / \mathrm{cm}^{3}\right)$ & $\mu\left(\mathrm{cm}^{2} / \mathrm{V} \mathrm{s}\right)$ & $n$ & $k(\mathrm{~W} / \mathrm{cm} \mathrm{K})$ \\
\hline $\mathrm{p}-\mathrm{GaN}$ (contact) & 30 & $2 \times 10^{20}$ & 10 & 2.54 & 1.3 \\
\hline $\mathrm{p}-\mathrm{Al}_{0.14} \mathrm{Ga}_{0.86} \mathrm{~N} / \mathrm{GaN} \mathrm{SL}$ (cladding) & 600 & $1 \times 10^{20}$ & 0.5 & 2.48 & 0.2 \\
\hline p-GaN (waveguide) & 100 & $5 \times 10^{18}$ & 15 & 2.54 & 1.3 \\
\hline $\mathrm{p}-\mathrm{Al}_{0.2} \mathrm{Ga}_{0.8} \mathrm{~N}$ (stopper) & 20 & $1 \times 10^{19}$ & 10 & 2.23 & 0.8 \\
\hline $\mathrm{n}-\mathrm{In}_{0.02} \mathrm{Ga}_{0.98} \mathrm{~N}$ (barrier) & 10 & $7 \times 10^{16}$ & 850 & 2.61 & 0.2 \\
\hline $\mathrm{n}$-In $\mathrm{n}_{0.15} \mathrm{Ga}_{0.85} \mathrm{~N}$ (quantum well) & 4 & $7 \times 10^{16}$ & 300 & 3.0 & 0.2 \\
\hline $\mathrm{n}-\mathrm{In}_{0.02} \mathrm{Ga}_{0.98} \mathrm{~N}$ (barrier) & 10 & $7 \times 10^{16}$ & 850 & 2.61 & 0.2 \\
\hline $\mathrm{n}$-In $\mathrm{n}_{0.15} \mathrm{Ga}_{0.85} \mathrm{~N}$ (quantum well) & 4 & $7 \times 10^{16}$ & 300 & 3.0 & 0.2 \\
\hline $\mathrm{n}-\mathrm{In}_{0.02} \mathrm{Ga}_{0.98} \mathrm{~N}$ (barrier) & 10 & $7 \times 10^{16}$ & 850 & 2.61 & 0.2 \\
\hline n-GaN (waveguide) & 100 & $7 \times 10^{17}$ & 550 & 2.54 & 1.3 \\
\hline $\mathrm{n}-\mathrm{Al}_{0.14} \mathrm{Ga}_{0.86} \mathrm{~N} / \mathrm{GaN} \mathrm{SL}$ (cladding) & 1200 & $3 \times 10^{18}$ & 10 & 2.48 & 0.2 \\
\hline $\mathrm{n}$-In $\mathrm{n}_{0.1} \mathrm{Ga}_{0.9} \mathrm{~N}$ (compliance) & 100 & $3 \times 10^{18}$ & 390 & 2.98 & 1.0 \\
\hline n-GaN (substrate) & 3000 & $3 \times 10^{18}$ & 410 & 2.54 & 1.3 \\
\hline
\end{tabular}

Table 1: Layer structure and room-temperature parameters of nitride laser

$I=$ layer thickness, $N_{\text {dop }}=$ doping, $\mu=$ majority carrier mobility (low field), $n=$ refractive index ( 400 nm wavelength), $k=$ thermal conductivity 


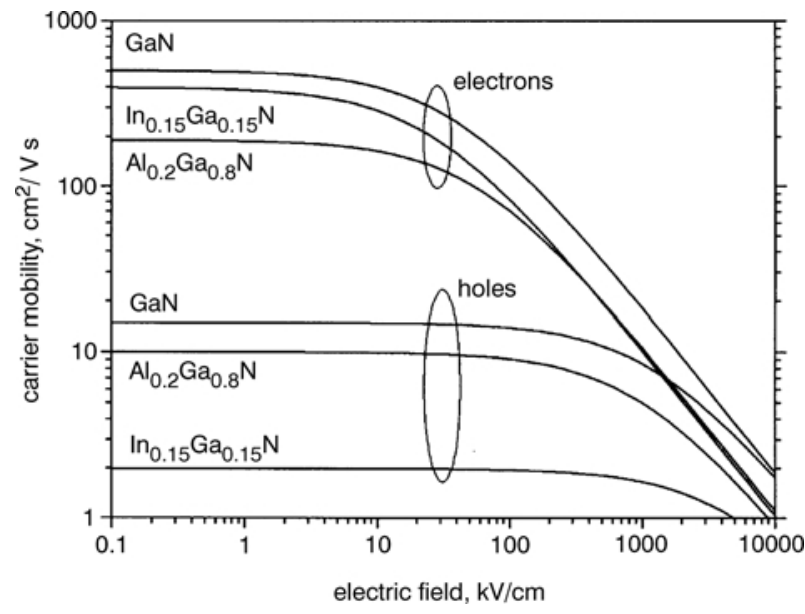

Fig. 2 Mobility of electrons and holes as function of electrostatic field for bulk nitride alloys

At room temperature, with $10^{18} \mathrm{~cm}^{-3}$ carrier concentration

of phonons which is known to strongly reduce the thermal conductivity.

\section{Comparison to measurements}

Our parameters of the drift-diffusion model give good agreement with the measured current-voltage (IV) characteristic (Fig. 6). To find simultaneous agreement with the measured light-current (LI) characteristic, three fit parameters are employed which are unknown for the present device: the internal modal loss $\alpha_{i}$, the defect (SRH) recombination lifetime within the quantum wells $\tau_{q w}$ and the thermal resistance $R_{t h}$. The modal loss mainly controls the slope efficiency, the lifetime affects the threshold

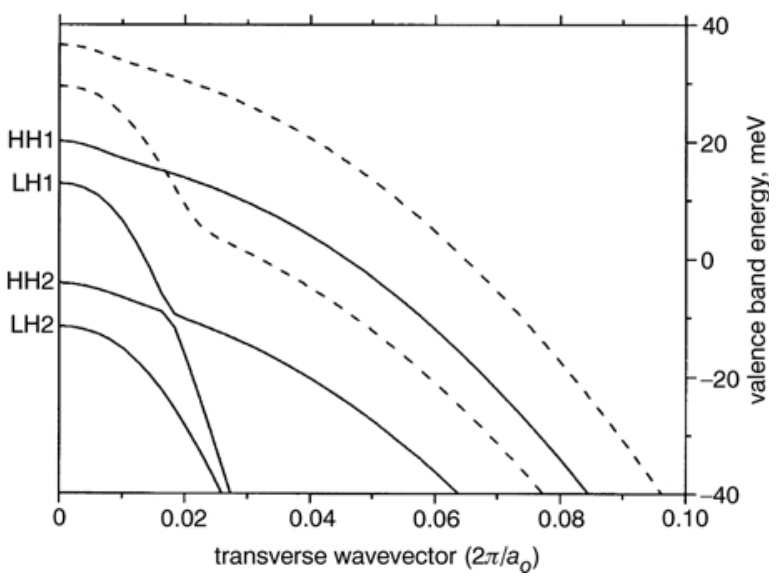

Fig. 3 Valence band structure of our quantum wells

$\mathrm{HH}$, heavy hole bands; LH, light hole bands; solid lines, $1.6 \%$ compressive strain; dashed lines upper two bands without strain

current, and thermal resistance has a major impact on the power roll-off. The values $\alpha_{i}=12 \mathrm{~cm}^{-1}, \tau_{q w}=0.5 \mathrm{~ns}$ and $R_{t h}=75 \mathrm{~K} / \mathrm{W}$ result in an excellent reproduction of the experimental LI curve (Fig. 6). All three numbers are reasonable and confirm the accuracy of the laser model. In further agreement with the experimental observation [1], a first order lateral laser mode emerges in the simulation, besides the fundamental mode (Figs. 6 and 7).

\section{Physical analysis}

The output power roll-off of laser diodes is typically attributed to self-heating in continuous-wave operation. The data in Fig. 6 indicate that $\sim 4 \mathrm{~W}$ total heat power is

Table 2: Electron bandstructure parameters for nitride wurtzite semiconductors at room temperature

\begin{tabular}{|c|c|c|c|c|c|}
\hline Parameter & Symbol & Unit & $\ln N$ & $\mathrm{GaN}$ & AIN \\
\hline Electron eff. mass (c-axis) & $m_{c}^{z}$ & $m_{0}$ & 0.11 & 0.20 & 0.33 \\
\hline Electron eff. mass (transversal) & $m_{c}^{t}$ & $m_{0}$ & 0.11 & 0.18 & 0.25 \\
\hline Hole eff. mass parameter & $A_{1}$ & - & -9.24 & -7.24 & -3.95 \\
\hline Hole eff. mass parameter & $A_{2}$ & - & -0.60 & -0.51 & -0.27 \\
\hline Hole eff. mass parameter & $A_{3}$ & - & 8.68 & 6.73 & 3.68 \\
\hline Hole eff. mass parameter & $A_{4}$ & - & -4.34 & -3.36 & -1.84 \\
\hline Hole eff. mass parameter & $A_{5}$ & - & -4.32 & -3.35 & -1.92 \\
\hline Hole eff. mass parameter & $A_{6}$ & - & -6.08 & -4.72 & -2.91 \\
\hline Direct bandgap & $E_{g}$ & $\mathrm{eV}$ & 1.89 & 3.42 & 6.28 \\
\hline Spin-orbit energy & $\Delta_{\text {so }}$ & $\mathrm{eV}$ & 0.001 & 0.013 & 0.019 \\
\hline Crystal-field energy & $\Delta_{c r}$ & $\mathrm{eV}$ & 0.041 & 0.042 & -0.217 \\
\hline Lattice constant & $a_{o}$ & $\AA$ & 3.548 & 3.189 & 3.112 \\
\hline Elastic constant & $C_{33}$ & $\mathrm{GPa}$ & 200 & 392 & 382 \\
\hline Elastic constant & $C_{13}$ & $\mathrm{GPa}$ & 94 & 100 & 127 \\
\hline Hydrost. deform. potential (interband) & a & $\mathrm{eV}$ & & -8.16 & \\
\hline Hydrost. deform. potential $\left(E_{c}\right)$ & $a_{c}$ & $\mathrm{eV}$ & & -4.08 & \\
\hline Shear deform. potential & $D_{1}$ & $\mathrm{eV}$ & & -0.89 & \\
\hline Shear deform. potential & $D_{2}$ & $\mathrm{eV}$ & & 4.27 & \\
\hline Shear deform. potential & $D_{3}$ & $\mathrm{eV}$ & & 5.18 & \\
\hline Shear deform. potential & $D_{4}$ & $\mathrm{eV}$ & & -2.59 & \\
\hline
\end{tabular}

$\Delta_{c r}=\Delta_{1}, \Delta_{s o}=3 \Delta_{2}=3 \Delta_{3}[9,11,14,15,17,25]$ 


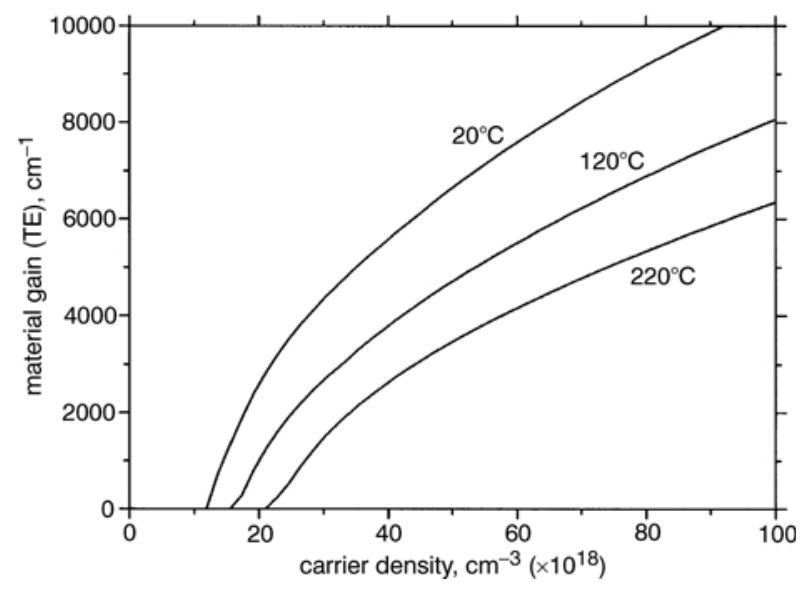

Fig. 4 Gain against carrier density for our quantum wells at different temperatures

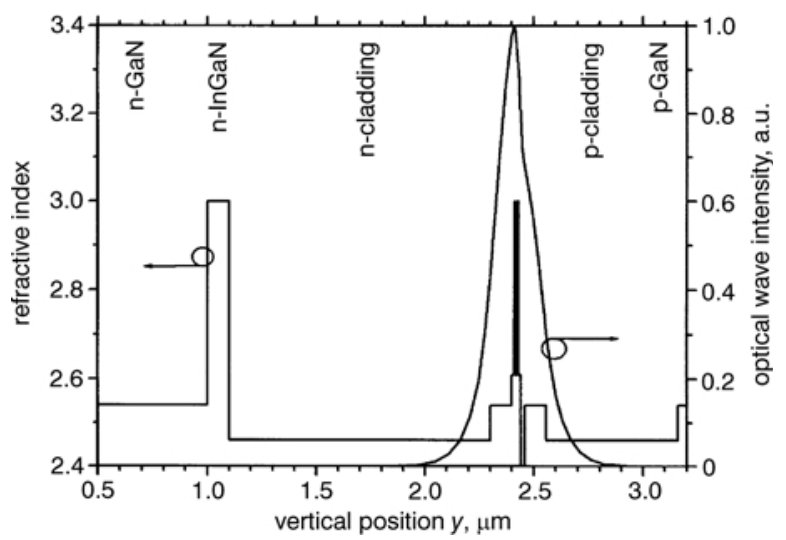

Fig. 5 Vertical profile of refractive index and optical intensity at laser axis

generated at the output power maximum. Considering $75 \mathrm{~K} / \mathrm{W}$ thermal resistance, the internal temperature rise can be estimated as $\Delta T=300 \mathrm{~K}$. The contour plot of the calculated temperature distribution $T(x, y)$ confirms this strong self-heating (Fig. 8). Within the centre part of the laser, the thermal model employs the thermal conductivity values given in Table 1 . The rest of the substrate and the heat sink are represented by an external thermal resistance which is used as a fit parameter, contributing $30 \mathrm{~K} / \mathrm{W}$ to the total thermal resistance $R_{t h}=75 \mathrm{~K} / \mathrm{W}$. Eliminating this

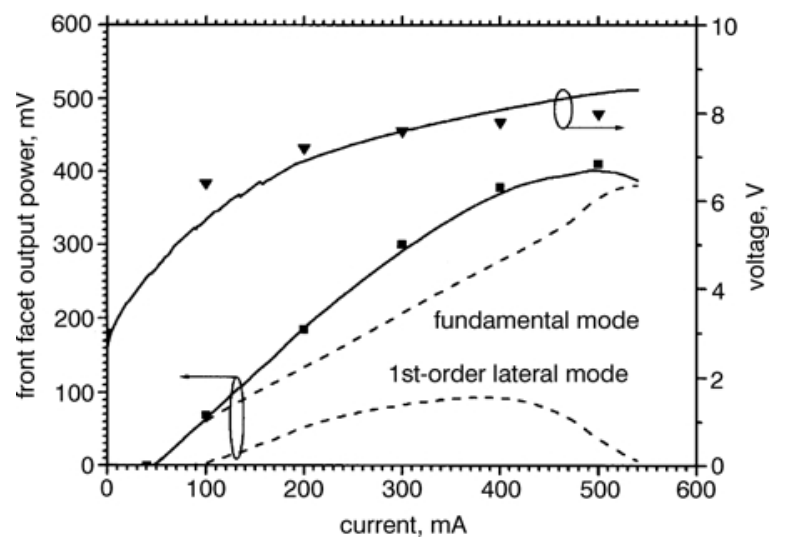

Fig. 6 Current-voltage and light-current characteristics: comparison of simulation and measurement

Lines, simulation; points, measured values

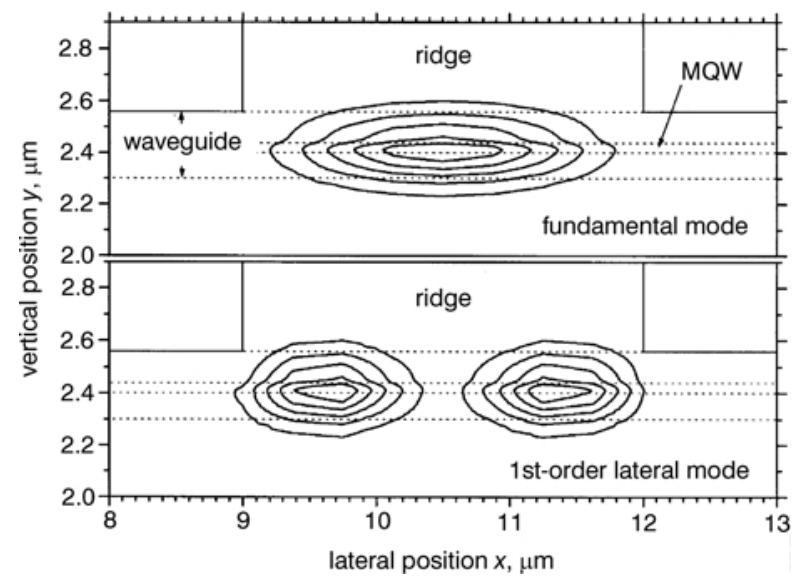

Fig. 7 2-D profile of the two lasing modes

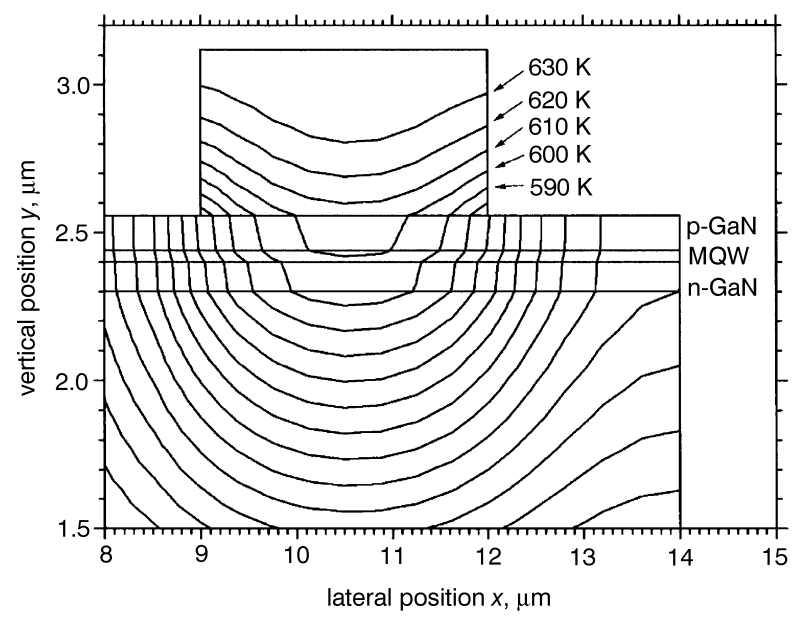

Fig. 8 Internal temperature distribution $T(x, y)$ at maximum output power

external thermal resistance will be shown to have a significant impact on the maximum output power (Section 6). The different heat sources are illustrated in Fig. 9. Joule heat in the highly resistive p-doped regions dominates the power budget by far. The much smaller contribution from phonons generated by defect recombination is partially compensated for by Thomson cooling, which represents the phonon absorption by carriers, e.g. during thermionic

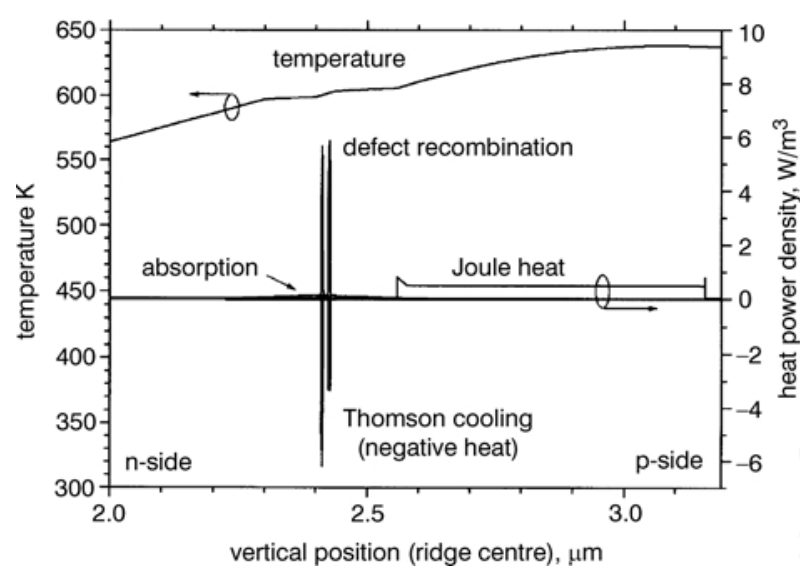

Fig. 9 Heat source profile and temperature profile at maximum output power 


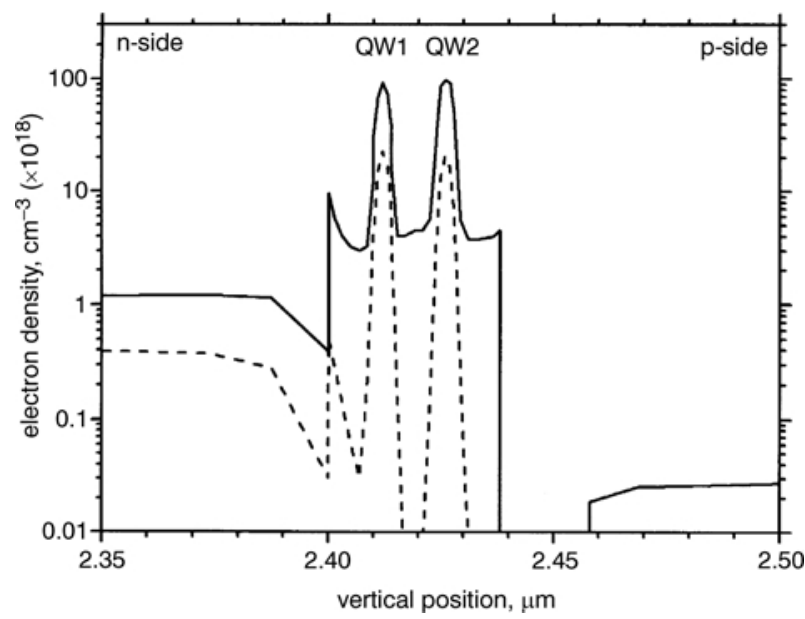

Fig. 10 Vertical profile of electron density at threshold and at maximum power

Dashed lines, threshold; solid lines, maximum power

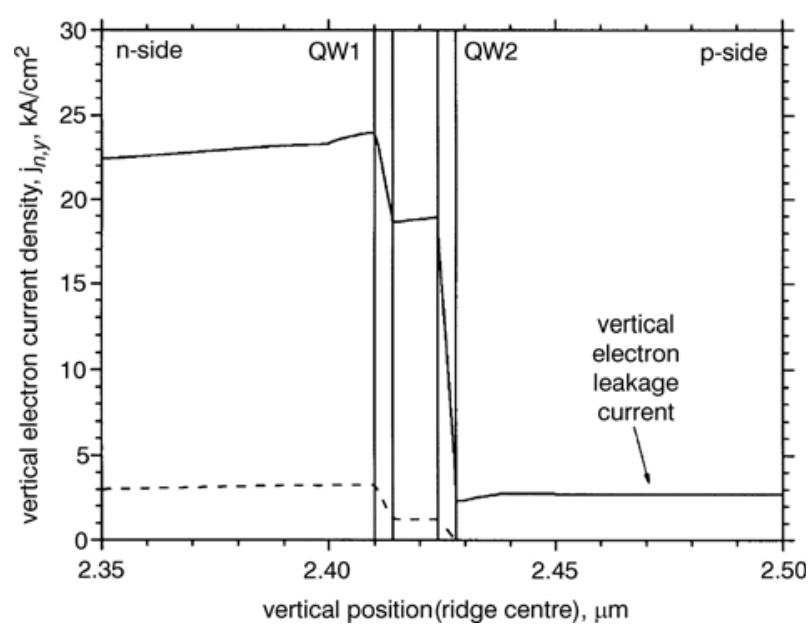

Fig. 11 Vertical electron current at laser axis Dashed lines, threshold; solid lines, maximum power

emission from the quantum wells. Heat from photon absorption is of minor importance.

The strong self-heating reduces the optical gain substantially (Fig. 4), which is mainly caused by a wider Fermi spreading of carriers. Consequently, the carrier density increases with higher temperature (Fig. 10). The quantum well electron density is $\sim 2 \times 10^{19} \mathrm{~cm}^{-3}$ at threshold but it is substantially higher at the power maximum. This leads to enhanced recombination losses but, most of all, to an escalation of electron leakage from the quantum wells into the p-side. Fig. 11 plots the vertical electron current profile. At threshold, all electrons recombine within the quantum wells and no electron leaks into the p-side. At maximum power, a considerable portion of electrons escapes across the AlGaN stopper layer. This vertical electron leakage amounts to more than one-third of the total carrier losses at the power maximum. As it prevents carriers from stimulated recombination, it is the main reason for the power roll-off.

\section{Polarisation effects}

Spontaneous polarisation as well as strain-induced polarisation in nitride compounds results in polarisation charges at hetero interfaces and in built-in polarisation fields. We

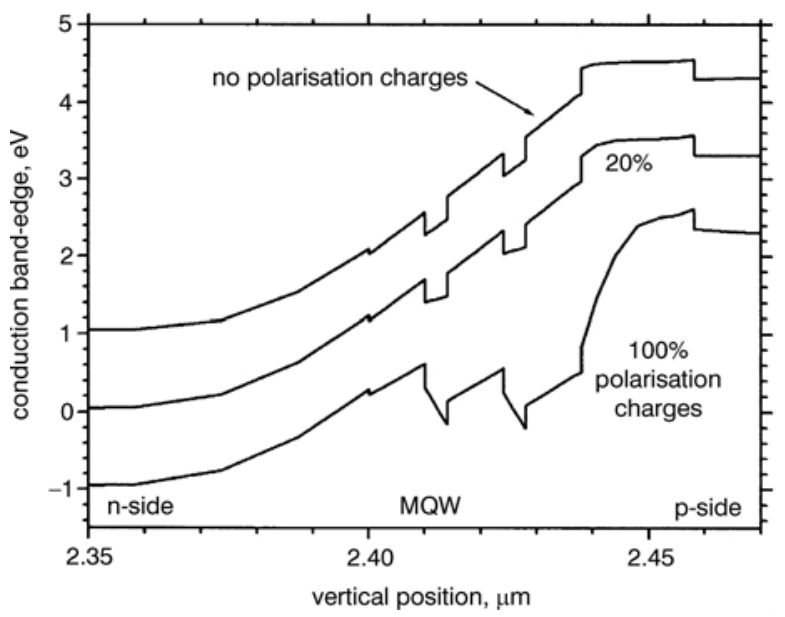

Fig. 12 Conduction band-edge at laser axis for 0, 20 and 100\% polarisation charges at zero bias

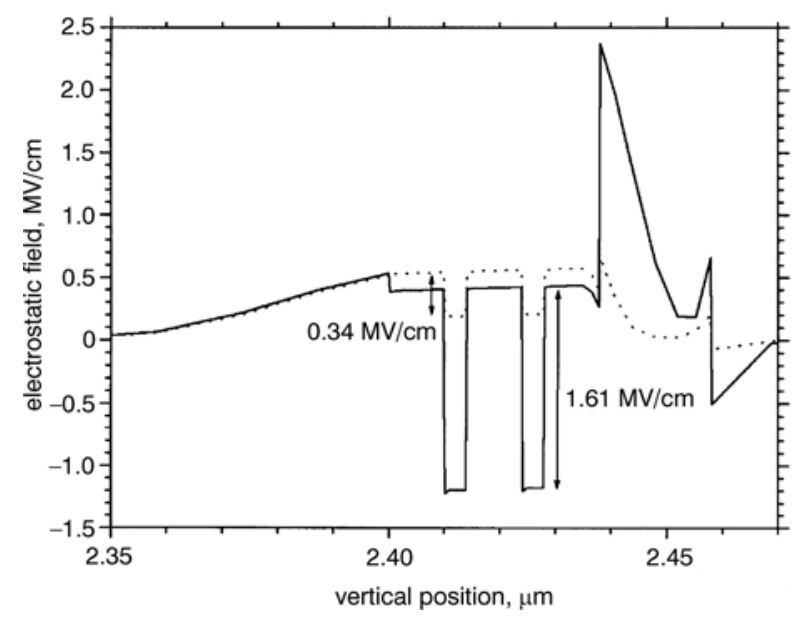

Fig. 13 Vertical component of electrostatic field at laser axis for $20 \%$ and for $100 \%$ polarisation charges

Dotted lines, 20\%; solid lines, 100\%

calculate these charges by linear interpolation of binary material parameters given in [26]. Their effect on the conduction band-edge and on the electrostatic field at zero bias is shown in Figs. 12 and 13, respectively. Full consideration of the calculated polarisation charges results

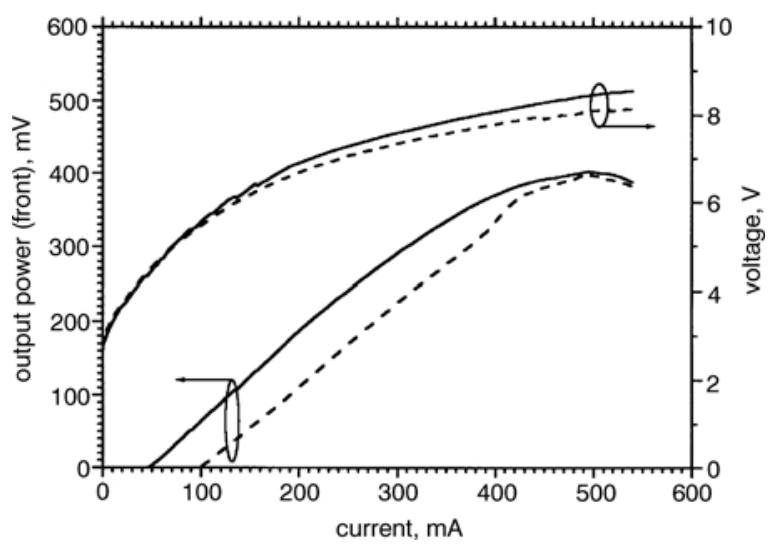

Fig. 14 Light-current and current-voltage characteristics without polarisation and with $20 \%$ polarisation charges

Solid lines, no polarisation; dashed lines, $20 \%$ polarisation 
in a strong deformation of the QW potential by the built-in QW polarisation field of $1.61 \mathrm{MV} / \mathrm{cm}$. However, experimental investigations give a variety of polarisation field strengths. This is mainly attributed to charged defects at hetero interfaces which partially screen the polarisation field. The actual polarisation field in our quantum wells is unknown and we assume $20 \%$ of the calculated polarisation charges corresponding to a $340 \mathrm{kV} / \mathrm{cm}$ QW polarisation field, which is close to experimental results on similar quantum wells [27]. This assumption doubles the calculated threshold current due to quantum well gain reduction by the separation of electron and hole wave functions (Fig. 14). However, the rising carrier density with higher lasing power leads to enhanced screening of the polarisation charges. The maximum output power is the same with or without polarisation. Therefore, polarisation effects are neglected in the rest of the paper.

\section{Laser optimisation}

Reduction of self-heating seems to be the key to higher maximum output power. This can be achieved by lowering the heat power generation or by improved heat dissipation. The electrical resistance of the p-doped layers constitutes the main heat source but it cannot be easily reduced since higher p-doping causes a lower hole mobility. Improved heat sinking has recently been demonstrated by replacing the sapphire substrate with copper [28]. In our simulation we simply eliminate the external thermal resistance, which reduces the total thermal resistance from $75 \mathrm{~K} / \mathrm{W}$ to $45 \mathrm{~K} / \mathrm{W}$. The resulting $L I$ characteristic exhibits almost double the maximum output power (Fig. 15). The QW temperature at maximum power is about the same as that of the original laser.

Suppression of electron leakage may be achieved by using an AlGaN stopper layer with larger bandgap. Such a situation is simulated assuming an AlGaN electron stopper layer with $25 \%$ aluminium. The larger conduction band offset results in a slight increase of the maximum output power (Fig. 15). This is due to reduced electron leakage since all other material parameters of the stopper layer remained unchanged. However, thermal and electrical conductivities decrease with higher Al content, which is expected to counteract this improvement.

Anti-reflection coating of the front facet $\left(R_{f}=0.02\right)$ gives a slightly higher maximum power (Fig. 15). A

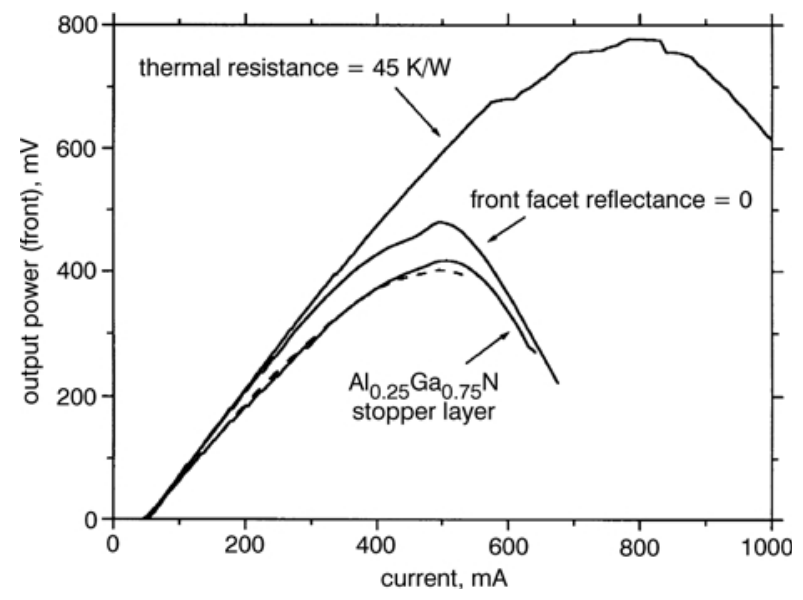

Fig. 15 LI characteristics resulting from laser improvements Dashed line indicates original characteristic wider ridge would substantially reduce the self-heating; however, the higher aspect ratio of the far-field as well as the enhanced probability of higher order lasing modes are not desirable. The influence of the number of quantum wells was previously investigated, and both experiment [29] and theory [30] find that two quantum wells is optimum.

\section{Summary}

Advanced device simulation is employed to investigate the internal physics of high-power nitride laser diodes. Measurements are used to confirm the validity of the laser model and to calibrate material parameters. By reproducing experimental laser characteristics in the simulation, quantitative links between microscopic physical processes and measured device performance are established. We reveal the dominating physical mechanisms in the causality chain that limits the maximum output power. The low electrical conductivity of p-doped layers leads to strong Joule heating, which reduces the optical gain in the quantum wells. As a consequence, the QW carrier density strongly increases, which results in enhanced carrier losses. Vertical electron leakage into the $\mathrm{p}$-doped region is the main cause for the power roll-off measured. Improved heat sinking is found to be most effective in maximising the output power.

\section{References}

1 NAKAMURA, S., SENOH, M., NAGAHAMA, S.I., IWASA, N., YAMADA, T., MATSUSHITA, T., KIYOKU, H., SUGIMOTO, Y., KOZAKI, T., UMEMOTO, I., SANO, M., and CHOCHO, K.: 'Violet InGaN/GaN/AlGaN-based laser diodes with an output power of 420 mW', Jpn. J. Appl. Phys. 2, Lett., 1998, 37, pp. L627-L629

2 LASTIP 6.1.1. Crosslight Software, 2001

3 GÖTZ, W., JOHNSON, N.M., WALKER, J., BOUR, D.P., and STREET, R.A.: 'Activation of acceptors in Mg-doped $\mathrm{GaN}$ grown by metalorganic chemical vapor deposition', Appl. Phys. Lett., 1996, 68, pp. 667-669

4 GÖTZ, W., JOHNSON, N.M., CHEN, C., LIU, H., KUO, C., and IMLER, W.: 'Activation energies of Si donors in GaN', Appl. Phys. Lett., 1996, 68, pp. 3144-3146

5 NAKAMURA, S., MUKAI, T., and SENOH, M.: 'In situ monitoring and Hall measurements of GaN grown with GaN buffer layers', J. Appl. Phys., 1992, 71, pp. 5543-5549

6 FARAHMAND, M., GARETTO, C., BELLOTTI, E., BRENNAN, K.F., GOANO, M., GHILliNO, E., GHIONE, G., ALBRECHT, J.D., and RUDEN, P.P.: 'Monte Carlo simulation of electron transport in the IIInitride wurtzite phase material system: binaries and ternaries', IEEE Trans. Electron Devices, 2001, 48, pp. 535-542

7 SOTOODEH, M., KHALID, A.H., and REZAZADEH, A.A.: 'Empirical low-field mobility model for III-V compounds applicable in device simulation', J. Appl. Phys., 2000, 87, (6), pp. 2890-2900

8 DMITRIEV, A.V., and ORUZHEINIKOV, A.L.: 'The rate of radiative recombination in the nitride semiconductors and alloys', MRS Internet J. Nitride Semicond. Res., 1996, 1, pp. 46

9 WETZEL, C., TAKEUCHI, T., YAMAGUCHI, S., KATOH, H., AMANO, H., and AKASAKI, I.: 'Optical band gap in GaInN on GaN by photoreflection spectroscopy', Appl. Phys. Lett., 1998, 73, pp. 1994-1996

10 LEE, S.R., WRIGHT, A.F., CRAWFORD, M.H., PETERSEN, G.A., HAN, J., and BIEFELD, R.M.: 'The band-gap bowing of $\mathrm{Al}_{x} \mathrm{Ga}_{1-x} \mathrm{~N}$ alloys', Appl. Phys. Lett., 1999, 74, pp. 3344-3346

11 WEI, S.-H., and ZUNGER, A.: 'Valence band spittings and band offsets of AlN, GaN, and InN', Appl. Phys. Lett., 1996, 69, pp. 2719-2721

12 ABARE, A.C.: 'Growth and fabrication of nitride-based distributed feedback laser diodes'. PhD thesis, University of California at Santa Barbara, 2000

13 CHUANG, S.L., and CHANG, C.S.: 'k.p method for strained wurtzite semiconductors', Phys. Rev. B, 1996, 54, pp. 2491-2504

14 CHUANG, S.L., and CHANG, C.S.: 'A band-structure model of strained quantum-well wurtzite semiconductors', Semicond. Sci. Technol., 1997, 12, pp. 252-263

15 YEO, Y.C., CHONG, T.C., LI, M.F., and FAN, W.J.: 'Analysis of optical gain and threshold current density of wurtzite InGaN/GaN/AlGaN quantum well lasers', J. Appl. Phys., 1998, 83, pp. 1813-1819

16 YEO, Y.C., CHONG, T.C., and LI, M.F.: 'Uniaxial strain effect on the electronic and optical properties of wurtzite $\mathrm{GaN}-\mathrm{AlGaN}$ quantum-well lasers', IEEE J. Quantum Electron., 1998, 34, pp. 2224-2232

17 STRITE, S., and MORKOC, H.: 'GaN, AlN, and InN: A review', J. Vac. Sci. Technol., 1992, 10, (4), pp. 1237-1266 
18 PARK, S.H., and CHUANG, S.L.: 'Many-body optical gain of wurtzite GaN-based quantum-well lasers and comparison with experiment', Appl. Phys. Lett., 1998, 72, pp. 287-289

19 PARK, S.H., and CHUANG, S.L.: 'Piezoelectric effects on electrical and optical properties of wurtzite GaN/AlGaN quantum well lasers', Appl. Phys. Lett., 1998, 72, pp. 3103-3105

$20 \mathrm{CHOW}$, W.W, and $\mathrm{KOCH}$, S.W. 'Theory of laser gain in group-III nitride quantum wells' in PEARTON, S.J. (Ed.): 'GaN and related materials' (Gordon \& Breach, New York, 1999), pp. 235-262

21 CHUANG, S.L.: 'Optical gain of strained wurtzite GaN quantum well lasers', IEEE J Quantum Electron , 1996, 32, (10), pp. 1791-1799

22 ADACHI, S.: 'Physical properties of III-V semiconductor compounds' (Wiley, New York, 1992)

23 LAWS, G.M., LARKINS, E.C., HARRISON, I., MOLLOY, C., and SOMERFORD, D.: 'Improved refractive index formulas for the AlGaN and InGaN alloys', J. Appl. Phys., 2001, 89, pp. 1108-1115

24 PIPREK, J., PENG, T., QUI, G., and OLOWOLAFE, J.O.: 'Energy gap bowing and refractive index spectrum of AlInN and AlGaInN'. Proceedings of IEEE International Symposium on Compound semiconductors, San Diego, CA, USA, 8-11 September, 1997, pp. 227-230

25 LEVINSHTEIN, M.E, RUMYANTSEV, S.L., and SHUR, M S. (Eds.) 'Properties of advanced semi-conductor materials' (Wiley, New York, 2001)
26 BERNARDINI, F., FIORENTINI, V., and VANDERBILT, D.: 'Accurate calculation of polarization-related quantities in semiconductors', Phys. Rev. B, 2001, 63, pp. 193-201

27 CHICHIBU, S.F., ABARE, A.C., MINSKY, M.S., KELLER, S., FLEISCHER, S.B., BOWERS, J.E., HU, E., MISHRA, U.K., COLDREN, L.A., and DENBAARS, S.P.: 'Effective band gap inhomogeneity and piezoelectric field in InGaN/GaN multiquantum well structures', Appl. Phys. Lett., 1998, 73, pp. 2006-2008

28 KNEISSL, M., WONG, W.S., TREAT, D.W., TEEPE, M., MIYASHITA, N., and JOHNSON, N.M.: 'Continuous-wave operation of InGaN multiple-quantum-well laser diodes on copper substrates obtained by laser liftoff', IEEE J. Sel. Topics Quantum Electron., 2001, 7, pp. 188-191

29 NAKAMURA, S., SENOH, M., NAGAHAMA, S.I., IWASA, N., YAMADA, T., MATSUSHITA, T., KIYOKU, H., SUGIMOTO, Y., KOZAKI, T., UMEMOTO, I., SANO, M., and CHOCHO, K. 'InGaN/GaN/AlGaN-based laser diodes grown on $\mathrm{GaN}$ substrates with a fundamental transverse mode', Jpn. J. Appl. Phys. 2, Lett., 1998, 37, pp. L1020-L1022

30 PIPREK, J., SINK, R.K., HANSEN, M.A., BOWERS, J.E., and DENBAARS, S.P.: 'Simulation and optimization of $420 \mathrm{~nm}$ InGaN/GaN laser diodes', Proc. SPIE-Int. Soc. Opt. Eng., 2000, 3944, pp. 28-39 